\title{
Time motion study to evaluate the impact of flattening filter free beam on overall treatment time for frameless intracranial radiosurgery using Varian TrueBeam ${ }^{\circledast}$ linear accelerator
}

\author{
Gopinath Mamballikalam ${ }^{1,3}$, Senthilkumar S. ${ }^{2}$, Clinto C.O. ${ }^{3}$, Ahamed Basith P.M. ${ }^{3}$, Jaon bos R.C. ${ }^{3}$, \\ Tems Thomas ${ }^{3}$ \\ ${ }^{1} \mathrm{R} \& \mathrm{D}$, Bharathiar University, Coimbatore, Tamilnadu, India \\ ${ }^{2}$ Department of Radiotherapy, Rajaji Hospital and Madurai Medical College, Madurai, Tamilnadu, India \\ ${ }^{3}$ Department of Radiation Oncology, Aster Medcity, Kochi, Kerala, India
}

\begin{abstract}
Background: The aim was to study the impact of the flattening filter free (FFF) beam on overall treatment time for frameless intracranial radiosurgery using TrueBeam ${ }^{\oplus}$ LINAC.

The development of frameless stereotactic radiosurgery (SRS) is possible due to the incorporation of image guidance in the delivery of treatment. It is important to analyze the cost and benefits of FFF beams for treating SRS by understanding the impact of FFF beams in reducing the treatment time.

Materials and methods: Dynamic conformal arc (DCA) and volumetric arc therapy (VMAT) plans were generated using 6 MV with a flattening filter (FF) and FFF beams. Overall treatment time was divided into beam on time (BOT) and beam off time (BFT). Percentage beam on time reduction (PBOTR) and Percentage total time reduction (PTTR) factors were defined for the comparison.

Results: BOT reduction was observed to be significant for higher dose per fraction but subjected to the treatment technique and modulation differences. PBOTR values are much higher than PTTR values. The $39.9 \%$ of PBOTR resulted in only $8 \%$ PTTR for DCA and $65.3 \%$ resulted in $15.9 \%$ PTTR for VMAT.

Conclusion: Major BFT was utilized for imaging and verification. FFF beam significantly reduced the beam on time and was found to be most effective if the fractional dose was as high as that for SRS. Newly defined PBOTR and PTTR factors are very useful indicators to evaluate the efficacy of FFF beams in terms of time reduction.
\end{abstract}

Key words: time motion study; flattening filter free; stereotactic radiosurgery; treatment time; frameless SRS

Rep Pract Oncol Radiother 2021;26(1):111-118

\section{Introduction}

Stereotactic radiosurgery (SRS) and Stereotactic Radiotherapy (SRT) utilizes a stereotactic apparatus and radiation beams to deliver a very high radiation dose to the lesion in single or multiple fractions. Stereotactic treatment techniques produce a concen- trated dose in the target with rapid dose fall off from the edge of the treated volume; it provides dramatic sparing of normal brain tissues [1]. SRS was first developed by Leksell in the late 1940s using orthovoltage X-rays. While dealing with small targets in intracranial radiosurgery, patient immobilization and treatment positioning accuracy is very significant.

Address for correspondence: Gopinath Mamballikalam MS.R.P., Department of Radiation Oncology, Aster Medcity, Kochi, Kerala, India, tel: (+91) 9656900749; e-mail: mgnmenon@gmail.com

This article is available in open access under Creative Common Attribution-Non-Commercial-No Derivatives 4.0 International (CC BY-NC-ND 4.0) license, allowing to download articles and share them with others as long as they credit the authors and the publisher, but without permission to change them in any way or use them commercially 
The traditional approach is to use an invasive head frame which can fix the cranium rigidly [2-4]. The drawbacks of using an invasive frame include patient anxiety, pain associated with the placement of the screws and risk of bleeding and infection at the site of placement. Noninvasive immobilization systems offer patient immobilization inferior to what is required for SRS, but sufficient and convenient for fractionated stereotactic radiotherapy. There were several publications on frame based and frameless radiosurgery [5-8]. To assure high precision and accuracy required for SRS when frameless noninvasive localization is used, the literature indicates that $3 \mathrm{D}$ image guidance is essential [9]. Non-invasive immobilization systems first proposed for linear accelerator and proton radiation technologies have now been developed for the Leksell Gamma Knife ICON radiosurgical system as well [10].

In conventional radiotherapy, targets are relatively large, so to provide large uniform X-ray fields a flattening filter was required. For small fields, a flattening filter is not necessary; by eliminating it dose rate can be enhanced. Modern LINAC provides a flattening filter-free beam with a higher dose rate. In MLC based Intensity Modulated Radiotherapy (IMRT) required fluence distribution can be achieved by modulating the un-flattened non-uniform fluence distribution directly [11]. The fluence of photons that comes out of an unflattened beam is dosimetrically different from a flattened beam $[12,13]$. Beam hardening does not occur since there is no flattening filter, and the fluence of photons contains a larger number of low energy photons $(<1 \mathrm{MeV})$. Head scatter and electron contamination are much lower since the flattening filter makes a significant contribution to it. The variation of the photon spectrum along the off-axis is much reduced in FFF beams. With an enhanced dose rate, beam on time will be reduced. But how much effect will it have on overall treatment time is dependent to the speed of the collimating system and image verification time [11].

In 2013, Florian Stieler et al. [14] carried out studies on Intensity Modulated radiosurgery (IMRS) with FFF beams and found that FFF radiosurgery is an efficient technique with reduced treatment time. In 2017, Thu M Dang et al. [15] assessed the efficacy of the FFF beam for stereotactic body radiation therapy (SBRT) and found that beam on time substantially reduced and appeared to impact the frequency of intra-fraction imaging, which reduced overall treatment time.
The development of frameless SRS is possible due to the incorporation of image guidance in the delivery of treatment. Single or hypo-fractionated treatment regime with the help of image guidance planning target volume (PTV) margins can be reduced significantly and results in excellent local control with minimal toxicity. These high precision radiotherapy techniques rely upon the imaging systems for immobilization, tumor positioning, and verification and the treatment time may be longer. Aim of this study to evaluate the impact of the FFF beam on overall treatment time for frameless intracranial radiosurgery.

\section{Materials and methods}

Varian TrueBeam STx linear accelerator with high definition $2.5 \mathrm{~mm}$ leaf width at isocenter multi leaf collimator (MLC) was used in this study. 6D couch, $\mathrm{kV}$ cone beam CT and BrianLAB Exac Trac ${ }^{\circledR}$ $\mathrm{X}$-ray imaging system were used to support high precision radiotherapy [17]. The Linac was calibrated to deliver $1 \mathrm{cGy} / \mathrm{MU}$ to water at Dmax for $10 \times 10 \mathrm{~cm}^{2}$ at SSD setup as per the recommendations of TRS-398 and TRS-483 [18, 19]. 6 MV FF $\left(\mathrm{TPR}_{20,10}: 0.669\right.$; Max dose rate: $\left.600 \mathrm{MU} / \mathrm{min}\right)$ and 6 MV FFF (TPR 20,10 : 0.632; Max dose rate: 1400 $\mathrm{MU} / \mathrm{min}$ ) beams were used. Dynamic conformal arc (DCA) and Intensity Modulated Radiosurgery (IMRS) plans were created with a single isocenter using the Eclipse treatment planning system. Anisotropic Analytical Algorithm (AAA) version 13.7.16 was used for dose calculation. All the plans had used five arcs for both DCA and IMRS by volumetric arc therapy (VMAT); consisted of two coplanar and three non-coplanar arcs. Patients were immobilized using BrainLAB frameless mask system. Congruence of CBCT and Exac Trac X-ray imaging isocenter had maintained below $0.3 \mathrm{~mm}$. PTV margin of $0.7 \mathrm{~mm}$ was given for SRS and 1.5 $\mathrm{mm}$ for SRT cases.

Overall treatment time was divided into Beam on time (BOT) and Beam off time (BFT). There are six sub-processes involved during BFT: 1. Initial positioning, 2. Initial imaging and verification, 3. Repositioning, 4. In-between couch motion, 5. In-between imaging and verification and 6. Releasing of the patient, Time devoted for each process was monitored using stop watch during patient treatment. To obtain BFT, 30 patient treatments 
were monitored with five patients for each dose per fraction of 5, 9, 12, 16, 20 and 25 Gy. For each dose per fraction four plans were created: DCA with FF, DCA with FFF, VMAT with FF and VMAT with FFF. BOT was monitored by the beams delivered on the phantom.

\section{Image guidance protocol}

After the immobilization and initial positing of the patient, ExacTrac X-ray imaging was done. Verification was done by registering acquired 2D image with digitally reconstructed radiograph (DRR). Repositioning of patient was required if shift found was above $0.7 \mathrm{~mm}$ by using BrainLAB $6 \mathrm{D}$ couch. A cone beam CT (CBCT) scan was acquired and verified with planning CT. If couch shifts were below $0.2 \mathrm{~mm}$, first section of couch zero arc would be proceeded. Before each non-coplanar arc at different couch angles, MV imaging would be done at zero gantry and Exactrac imaging at regular intervals during the treatment. At the end of the non-coplanar arcs, the CBCT scan would be acquired followed by the last section of couch zero arcs.

\section{Comparison indices}

Percentage beam on time reduction (PBOTR) and percentage total time reduction (PTTR) factors were defined for the comparison of flattened with unflattened plans. The higher the PBOTR and PTTR values, the better FFF beam in reducing beam on time and overall treatment time, respectively.

$$
\begin{aligned}
& \text { PBOTR }=\left(1-\frac{\text { BeamOn }_{F F F}}{\text { BeamOn }_{F F}}\right) \times 100 \% \\
& \text { PTTR }=\left(1-\frac{\text { TotalTime }_{F F F}}{\text { TotalTime }_{F F}}\right) \times 100 \%
\end{aligned}
$$

where: Beam $\mathrm{On}_{\mathrm{FFF}}-$ beam on time of unflattened plan; Beam $\mathrm{On}_{\mathrm{FF}}$ - beam on time of flatted plan;

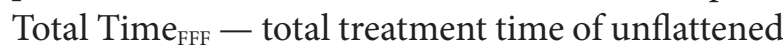
plan; Total Time $\mathrm{FF}_{\mathrm{FF}}$ - total treatment time of flattened plan.

PBOTR and PTTR of 6 MV DCA and VMAT plans for FF and FFF energies were estimated for six independent plans with 5, 9, 15, 16, 20 and 25 Gy dose per fraction.

\section{Time and motion study}

Time and motion study is a method of data collection that records the time taken for various movements as part of the process to perform a defined repetitive task [16]. The study consists of two parts wherein the first part involves capturing the time and its analysis results in knowing the time taken to accomplish a specified task and the other part relating to the motion denotes the various steps in the process that could be altered to achieve better efficiency in the process. Applicability of time and motion study in the service industry has brought about the importance of efficacy in the systems; resulting in a better opportunity cost of time. This method of study is based on the fundamental concept of the value of time and is a tool to maximize productivity. The study is also based on a few focal points, such as optimal allocation of resources to deliver the defined task, elimination of "Muda" and cost control measures. Eliminating "Muda", a Japanese term that means futility, is the main objective of the time and motion study. The idea is to optimize resources and increase satisfaction levels to the receiver of the service to achieve higher quality in the outcomes. Increased time in performing certain tasks means reduced productivity resulting in higher costs. Time and motion study enumerates the elements in the process. To control the elements in the process leads to reduced costs and increased outcomes. The potential areas of reducing human efforts are the core of the motion study through simplifying and minimizing manual efforts. The study begins with the understanding of the entire process and recording the activities with the precise time taken for the movements. To further improve understanding, each of the movements is studied to identify the areas that could be customized. Upon detailed analysis and customization of the steps in the process, the result obtained is the apt time and most efficient way of performing the specified task. The study is also a tool that aids in performing the evaluation and determination of the maximum output possible. With time fine-tuning, the process leads to establishing standards and bringing out best practices in the service industry specific to the task.

\section{Results}

Table 1 shows the minimum, maximum and average time devoted for each sub process involved 
Table 1. Minimum, maximum and average time devoted for each sub processes involved during beam off. Beam off time (BFT) is the sum of $\mathrm{t} 1, \mathrm{t} 2, \mathrm{t} 3, \mathrm{t} 4, \mathrm{t} 5$ and $\mathrm{t} 6$

\begin{tabular}{|c|c|c|c|c|c|c|c|}
\hline & $\begin{array}{l}\text { Initial } \\
\text { Positioning } \\
\text { time }\end{array}$ & $\begin{array}{l}\text { Initial } \\
\text { imaging and } \\
\text { verification } \\
\text { time }\end{array}$ & $\begin{array}{l}\text { Repositioning } \\
\text { time }\end{array}$ & $\begin{array}{l}\text { In-between } \\
\text { couch motion } \\
\text { time }\end{array}$ & $\begin{array}{l}\text { In-between } \\
\text { imaging and } \\
\text { verification } \\
\text { time }\end{array}$ & $\begin{array}{l}\text { Time for } \\
\text { releasing } \\
\text { patient }\end{array}$ & $\begin{array}{c}\text { Total Beam off } \\
\text { time }\end{array}$ \\
\hline & t1 & t2 & t3 & t4 & t5 & t6 & BFT \\
\hline Minimum & $3 \mathrm{~m}$ & $4 m$ & $1 \mathrm{~m}$ & $2 m$ & $3 \mathrm{~m}$ & $2 \mathrm{~m}$ & $15 \mathrm{~m}$ \\
\hline Average & $7 \mathrm{~m} 30 \mathrm{~s}$ & $6 \mathrm{~m}$ & $2 \mathrm{~m}$ & $3 \mathrm{~m}$ & $5 \mathrm{~m} 30 \mathrm{~s}$ & $3 \mathrm{~m}$ & $27 \mathrm{~m}$ \\
\hline Maximum & $9 \mathrm{~m}$ & $9 \mathrm{~m}$ & $4 \mathrm{~m}$ & $4 \mathrm{~m}$ & $8 \mathrm{~m}$ & $4 m$ & $38 \mathrm{~m}$ \\
\hline
\end{tabular}

$\mathrm{M}$ - minutes; $\mathrm{s}$ - seconds

Table2. Beam on time for dynamic conformal arc (DCA) and VMAT plans of 6 MV FF and FFF beams for different dose per fraction

\begin{tabular}{|c|c|c|c|c|}
\hline \multirow{3}{*}{ Dose/fr (Gy) } & \multicolumn{4}{|c|}{ Beam on time (BOT) } \\
\hline & \multicolumn{2}{|c|}{ DCA } & \multicolumn{2}{|c|}{ VMAT } \\
\hline & FF & FFF & FF & FFF \\
\hline 5 & $3 \mathrm{~m} 20 \mathrm{~s}$ & $3 \mathrm{~m} \mathrm{12s}$ & $2 \mathrm{~m} 20 \mathrm{~s}$ & $1 \mathrm{~m} 49 \mathrm{~s}$ \\
\hline 9 & $4 \mathrm{~m} 28 \mathrm{~s}$ & $3 \mathrm{~m} 50 \mathrm{~s}$ & $5 \mathrm{~m} 45 \mathrm{~s}$ & $3 \mathrm{~m} 16 \mathrm{~s}$ \\
\hline 12 & $5 \mathrm{~m} 37 \mathrm{~s}$ & $3 \mathrm{~m} 36 \mathrm{~s}$ & $5 \mathrm{~m} 41 \mathrm{~s}$ & $3 \mathrm{~m} 14 \mathrm{~s}$ \\
\hline 16 & $5 \mathrm{~m} 22 \mathrm{~s}$ & $3 \mathrm{~m} 39 \mathrm{~s}$ & $8 \mathrm{~m} 41 \mathrm{~s}$ & $3 \mathrm{~m} 1 \mathrm{~s}$ \\
\hline 20 & $6 \mathrm{~m} 26 \mathrm{~s}$ & $3 \mathrm{~m} 54 \mathrm{~s}$ & $11 \mathrm{~m} 54 \mathrm{~s}$ & $5 \mathrm{~m} 36 \mathrm{~s}$ \\
\hline 25 & $6 \mathrm{~m} 46 \mathrm{~s}$ & $4 \mathrm{~m} 4 \mathrm{~s}$ & $14 \mathrm{~m} 34 \mathrm{~s}$ & $6 \mathrm{~m} 7 \mathrm{~s}$ \\
\hline
\end{tabular}

$\mathrm{M}$ - minutes; s - seconds

during beam off. Imaging and verification process grabbed the majority of beam off time. Total BFT is the sum of $\mathrm{t} 1, \mathrm{t} 2, \mathrm{t} 3, \mathrm{t} 4, \mathrm{t} 5$ and $\mathrm{t} 6$. There maximum BFT duration was $38 \mathrm{~min}$, minimum was found to be $15 \mathrm{~min}$ and on average $27 \mathrm{~min}$ qas consumed for BFT. Beam on time for DCA and VMAT plan of $6 \mathrm{MV} \mathrm{FF}$ and FFF beams for different dose per fraction were compared in Table 2. Beam on time was reduced for unflattened beam as expected due to its higher dose rate obtained in the absence of a flattening filter. However, we found BOT reduction depends on the treatment technique used and modulation differences in plans. BOT reduction was observed to be significant for higher dose per fraction. For 25 Gy dose delivery, BOT reduction was $8 \mathrm{~min} 27 \mathrm{sec}$ for the VMAT technique whereas the reduction for the DCA plan was only 2 min $42 \mathrm{~s}$.

Figure 1 is the representation of time contribution of sub-processes to deliver 25Gy in single fraction for both DCA and VMAT plans using FF and FFF beams. Among all the four treatment options, on average of $36.25 \%$ contribution was taken for immobilization and positioning, $33.5 \%$ time was devoted for the entire imaging and verification processes, $8.75 \%$ for releasing the patient and beam on time is only $21.5 \%$. There was a $7 \%$ reduction in the beam on time for DCA and 17\% for the VMAT delivery technique due to the unflattened beam.

The percentage beam on time reduction (PBOTR) and percentage total time Reduction (PTTR) factors of $6 \mathrm{MV}$ DCA and VMAT plans for different doses per fraction are tabulated in Table 3. There was a maximum PBOTR of $65.3 \%$ among VMAT plans and $39.9 \%$ among DCA plans. PBOTR maximum value was not for the highest dose per fraction. The maximum PTTR of $8 \%$ and $20.3 \%$ belonged to the highest dose of $25 \mathrm{~Gy}$ in a single fraction for DCA and VMAT technique, respectively. $39.9 \%$ of PBOTR resulted in only $8 \%$ PTTR for DCA and $65.3 \%$ resulted in $15.9 \%$ PTTR for VMAT. The advantage of the reduction in the beam on time is not significantly utilized for the reduction in overall treatment time. PBOTR values are much higher as compared to PTTR values as shown in Table 3 for both the DCA and VMAT plans. PBOTR and PTTR factors are plotted against dose per fraction for DCA 
A

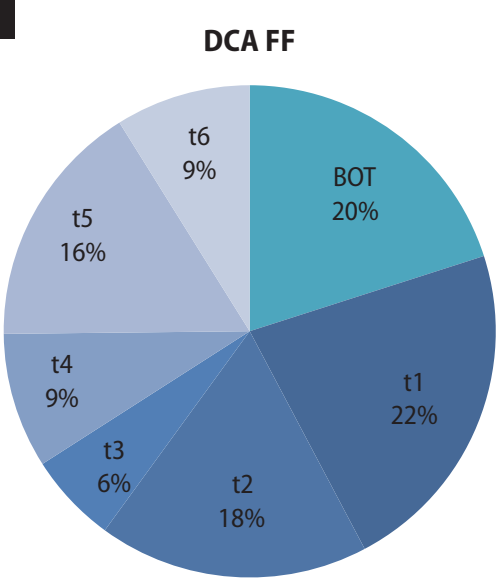

c

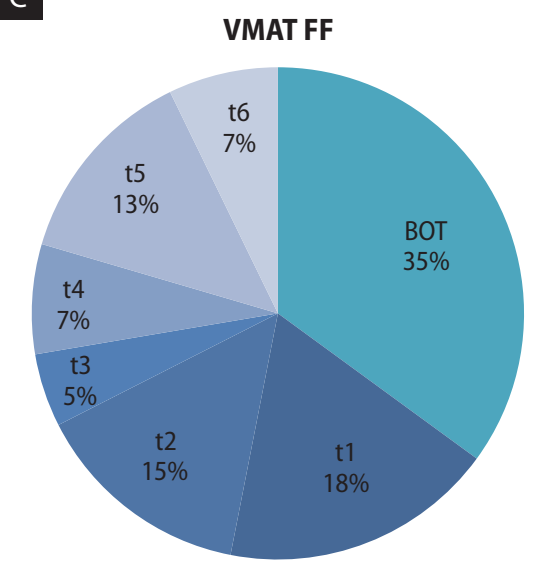

B

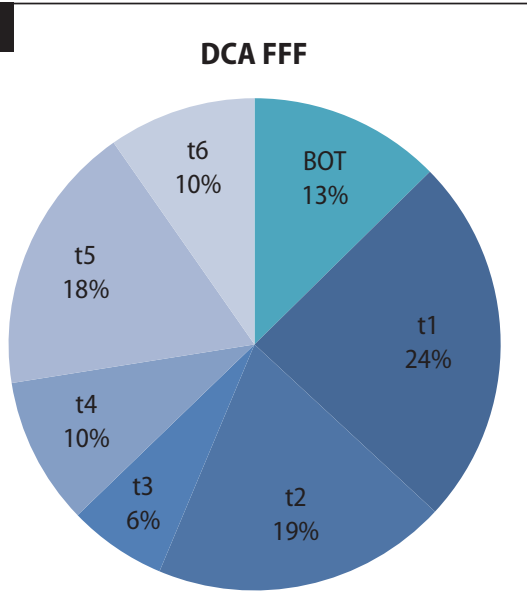

D

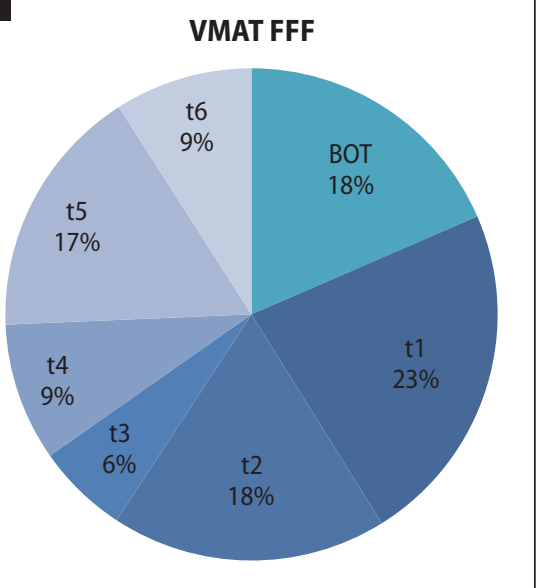

Figure 1. Contribution of average time taken for each sub process involved in beam off time and beam on time (BOT) to overall treatment time to deliver $25 \mathrm{~Gy}$. $\mathrm{t} 1$. Initial positioning time, $\mathrm{t} 2$. Initial imaging and verification time, $\mathrm{t} 3$. Repositioning time, $\mathrm{t} 4$. In-between couch motion time, $\mathrm{t} 5$. In-between imaging and verification time and $\mathrm{t} 6$. Time for releasing the patient. A. Dynamic conformal arc (DCA) plan with 6 MV FF beam; B. DCA plan with 6 MV FFF beam; C. Volumetric arc therapy (VMAT) plan with 6 MV FF beam; D. VMAT plan with 6 MV FFF beam

Table 3. Percentage beam on time reduction (PBOTR) and percentage total time reduction (PTTR) of $6 \mathrm{MV}$ dynamic conformal arc (DCA) and volumetric arc therapy (VMAT) plans for different dose per fraction

\begin{tabular}{|c|c|c|c|c|}
\hline \multirow{2}{*}{ Dose/Fr (Gy) } & \multicolumn{2}{|c|}{ PBOTR (\%) } & \multicolumn{2}{|c|}{ PTTR (\%) } \\
\hline & DCA & VMAT & DCA & VMAT \\
\hline 5 & 4.0 & 22.1 & 0.4 & 1.8 \\
\hline 9 & 14.2 & 43.2 & 2.0 & 7.6 \\
\hline 12 & 35.9 & 43.1 & 6.2 & 7.5 \\
\hline 16 & 32.0 & 65.3 & 5.3 & 15.9 \\
\hline 20 & 39.4 & 52.9 & 7.6 & 16.2 \\
\hline 25 & 39.9 & 58.0 & 8.0 & 20.3 \\
\hline
\end{tabular}

and VMAT techniques in Figure 2 and Figure 3. For the same dose per fraction VMAT plans had higher values of PBOTR and PTTR.

\section{Discussion}

Imaging and verification processes took the major time contribution to overall beam off time. It could be justified that the frameless SRS relies upon the image guidance system. Beam off time can be reduced by avoiding the second $\mathrm{CBCT}$ and also by modifying the imaging protocol. Beam on time for VMAT plan with flattening filter was longer than the DCA plan for a higher dose per fraction. But if the unfiltered beam was used for VMAT plans, the beam on time was significantly reduced. DCA plans did not have any intensity modulation, so there was a less substantial reduction in the beam on time at a higher dose per fraction.

The percentage beam on time reduction (PBOTR) and percentage total time reduction (PTTR) fac- 


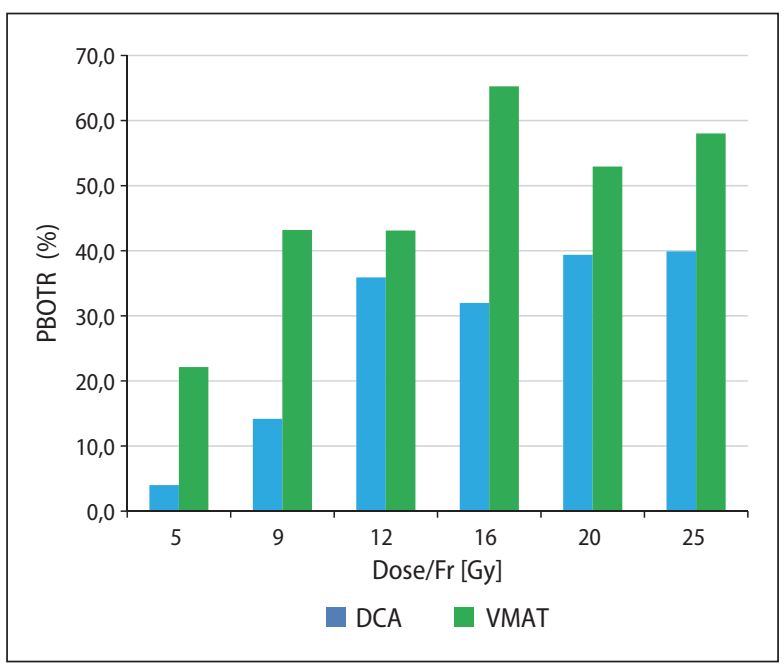

Figure 2. Percentage beam on time reduction (PBOTR) factor plotted against dose per fraction for dynamic conformal arc (DCA) and volumetric arc therapy (VMAT) techniques

tors were defined for evaluation. PBOTR values are much higher as compared to PTTR values for both the DCA and VMAT plans. For the same dose per fraction VMAT plans had higher values of PBOTR and PTTR. We observed that maximum PTTR was in the highest dose delivery for both the fluence un-modulated DCA and modulated VMAT. So, overall treatment time reduction is significant for high dose single fractions like SRS. Beam on time reduction depended on the delivery technique, degree of modulation and limitations of the collimating system.

It became very common to use the FFF beam for SRS/SRT. Several groups studied the difference between radiobiological properties of high dose rate FFF beam and conventional dose rate FF [20-22]. Currently, there is no evidence predicting clinically relevant effects of FFF beams at dose rates of up to $2500 \mathrm{MU} / \mathrm{min}$. With slower leaf speed, beam on time will be extended slightly longer [23]. As per the optimization constraints, mean dose rate and mean leaf speed determines the delivery time. Even with a higher dose rate, beam on time was delimited with mean MLC leaf speed (maximum leaf speed: $2.5 \mathrm{~cm} / \mathrm{s}$ ). As per JM Park et al. MLC speed and acceleration will affect the delivery accuracy of VMAT [24]. For SBRT, many studies recommended using FFF to minimize intra-fraction organ motion and frequency

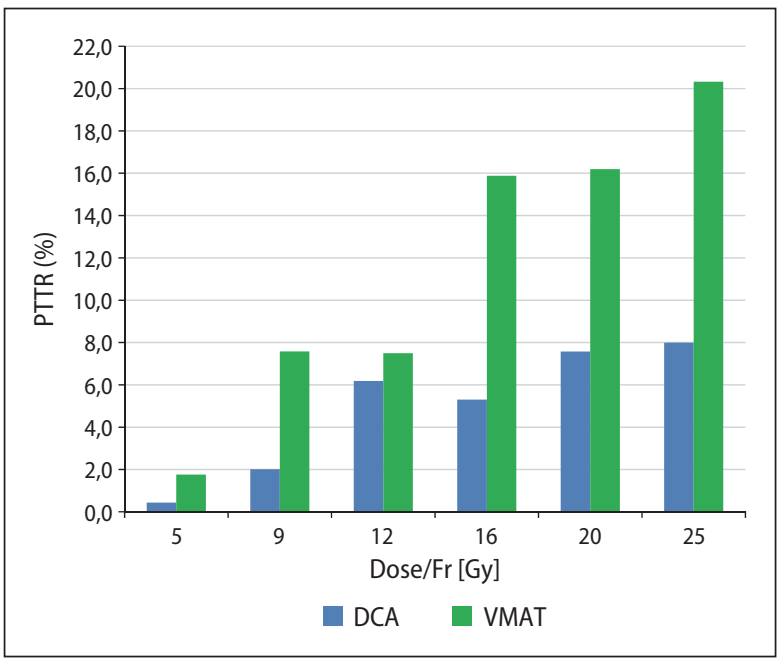

Figure 3. Percentage total time reduction (PTTR) factor plotted against dose per fraction for dynamic conformal arc (DCA) and volumetric arc therapy (VMAT) techniques

of imaging, reduce the patient breath-hold time for lung cases and, thus, to reduce overall treatment time [25-27]. But for intracranial radiosurgery with a frameless immobilization system, image guidance is inevitable and takes the longest process time as per time-motion study.

\section{Conclusion}

This study reveals interesting outcomes on how much FFF beam is useful in reducing overall treatment time for frameless intracranial radiosurgery. The increased dose rate of a FFF beam (2.3 times higher) does not necessarily directly translate into shorter treatment time. During overall treatment, beam off time is longer than beam on time. Major BFT is utilized for imaging and verification as frameless radiosurgery relies on image guidance. FFF beam significantly reduced the beam on time and was found to be most effective if the fractional dose was as high as that for SRS. Beam on time also depends on the treatment delivery technique and plan specific modulations due to the limitations of the collimating system. Newly defined PBOTR and PTTR factors are very useful indicators to evaluate the efficacy of the flattening filter free beams in terms of time reduction.

\section{Conflict of interest}

None declared. 


\section{Funding}

None declared.

\section{Acknowledgements}

Authors sincerely thank Mr. Sabrish K.G. for the support extended in the manuscript preparation.

\section{References}

1. Schell MC, Bova FJ, Larson DA. Stereotactic radiosurgery. American Association of Physicists in Medicine Report No. 54. American Institute of Physics, Woodbury 1995: 1-88.

2. Lightstone AW, Benedict SH, Bova FJ, et al. American Association of Physicists in Medicine Radiation Therapy Committee. Intracranial stereotactic positioning systems: Report of the American Association of Physicists in Medicine Radiation Therapy Committee Task Group No. 68. Med Phys. 2005; 32(7Part1): 2380-2398, doi: 10.1118/1.1945347, indexed in Pubmed: 28493584.

3. Lunsford LD, Leksell D. The Leksell System. In: Lunsford LD. ed. Modern Stereotactic Neurosurgery. Topics in neurological surgery. Vol 1. Springer, Boston 1988: MA.

4. Lutz W, Winston KR, Maleki N. A system for stereotactic radiosurgery with a linear accelerator. Int J Radiat Oncol Biol Phys. 1988; 14(2): 373-381, doi: 10.1016/03603016(88)90446-4, indexed in Pubmed: 3276655.

5. Ramakrishna N, Rosca F, Friesen S, et al. A clinical comparison of patient setup and intra-fraction motion using frame-based radiosurgery versus a frameless imageguided radiosurgery system for intracranial lesions. Radiother Oncol. 2010; 95(1): 109-115, doi: 10.1016/j. radonc.2009.12.030, indexed in Pubmed: 20116123.

6. Masi L, Casamassima F, Polli C, et al. Cone beam CT image guidance for intracranial stereotactic treatments: comparison with a frame guided set-up. Int J Radiat Oncol Biol Phys. 2008; 71(3): 926-933, doi: 10.1016/j. ijrobp.2008.03.006, indexed in Pubmed: 18514784.

7. Ruschin M, Nayebi N, Carlsson P, et al. Performance of a novel repositioning head frame for gamma knife perfexion and image-guided linac-based intracranial stereotactic radiotherapy. Int J Radiat Oncol Biol Phys. 2010; 78(1):306-313, doi: 10.1016/j.ijrobp.2009.11.001, indexed in Pubmed: 20385456.

8. Ali I, Tubbs J, Hibbitts K, et al. Evaluation of the setup accuracy of a stereotactic radiotherapy head immobilization mask system using kV on-board imaging. J Appl Clin Med Phys. 2010; 11(3): 3192, doi: 10.1120/jacmp.v11i3.3192, indexed in Pubmed: 20717086.

9. Babic S, Lee Y, Ruschin M, et al. To frame or not to frame? Cone-beam CT-based analysis of head immobilization devices specific to linac-based stereotactic radiosurgery and radiotherapy. J Appl Clin Med Phys. 2018; 19(2): 111-120, doi: 10.1002/acm2.12251, indexed in Pubmed: 29363282.

10. AIDahlawi I, Prasad D, Podgorsak MB. Evaluation of stability of stereotactic space defined by cone-beam CT for the Leksell Gamma Knife Icon. J Appl Clin Med Phys. 2017; 18(3): 67-72, doi: 10.1002/acm2.12073, indexed in Pubmed: 28419781.

11. Fu W, Dai J, Hu Y, et al. Delivery time comparison for intensity-modulated radiation therapy with/without flat- tening filter: a planning study. Phys Med Biol. 2004; 49(8): 1535-1547, doi: 10.1088/0031-9155/49/8/011, indexed in Pubmed: 15152690.

12. Mamballikalam G, Senthilkumar S, Jayadevan PM, et al. Evaluation of dosimetric parameters of small fields of $6 \mathrm{MV}$ flattening filter free photon beam measured using various detectors against Monte Carlo simulation. J Radiothera Pract. 2020: 1-1, doi: 10.1017/s1460396920000321.

13. Mamballikalam G, Senthilkumar S, Bos RCJ, et al. Stereotactic radiotherapy for small and very small tumours $(\leq 1$ to $\leq 3 \mathrm{cc}$ ): evaluation of the influence of volumetric-modulated arc therapy in comparison to dynamic conformal arc therapy and $3 \mathrm{D}$ conformal radiotherapy as a function of flattened and unflattened beam models. J Radiother Pract. 2020: 1-7, doi: 10.1017/s146039691900102x.

14. Stieler F, Fleckenstein J, Simeonova A, et al. Intensity modulated radiosurgery of brain metastases with flattening filter-free beams. Radiother Oncol. 2013; 109(3): 448-451, doi: 10.1016/j.radonc.2013.10.017, indexed in Pubmed: 24231243.

15. Dang TM, Peters MJ, Hickey B, et al. Efficacy of flatteningfilter-free beam in stereotactic body radiation therapy planning and treatment: A systematic review with meta-analysis. J Med Imaging Radiat Oncol. 2017; 61(3): 379-387, doi: 10.1111/1754-9485.12583, indexed in Pubmed: 28116813.

16. Lopetegui M, Yen PY, Lai A, et al. Time motion studies in healthcare: what are we talking about? J Biomed Inform. 2014;49:292-299, doi: 10.1016/j.jbi.2014.02.017, indexed in Pubmed: 24607863.

17. Gevaert T, Verellen D, Engels B, et al. Clinical evaluation of a robotic 6-degree of freedom treatment couch for frameless radiosurgery. Int J Radiat Oncol Biol Phys. 2012; 83(1):467-474, doi: 10.1016/j.jirobp.2011.05.048, indexed in Pubmed: 21945110.

18. Andreo P, Burns DT, Hohlfeld K. Absorbed dose determination in external beam radiotherapy: An international code of practice for dosimetry based on standards of absorbed dose to water. IAEA TRS-398, Vienna 2001.

19. IAEA, AAPM. Dosimetry of small static fields used in external beam radiotherapy: an international code of practice for reference and relative dose determination. Technical reports series no. 483. IAEA, Vienna 2017.

20. Ling CC, Gerweck LE, Zaider M, et al. Dose-rate effects in external beam radiotherapy redux. Radiother Oncol. 2010; 95(3): 261-268, doi: 10.1016/j.radonc.2010.03.014, indexed in Pubmed: 20363041.

21. Lohse I, Lang S, Hrbacek J, et al. Effect of high dose per pulse flattening filter-free beams on cancer cell survival. Radiother Oncol. 2011; 101(1): 226-232, doi: 10.1016/j. radonc.2011.05.072, indexed in Pubmed: 21733592.

22. Verbakel WF, van den Berg J, Slotman BJ, et al. Comparable cell survival between high dose rate flattening filter free and conventional dose rate irradiation. Acta Oncol. 2013; 52(3): 652-657, doi: 10.3109/0284186X.2012.7370 21, indexed in Pubmed: 23126524.

23. Kerns JR, Childress N, Kry SF. A multi-institution evaluation of MLC log files and performance in IMRT delivery. Radiat Oncol. 2014; 9: 176, doi: 10.1186/1748-717X-9-176, indexed in Pubmed: 25112533.

24. Park JM, Wu HG, Kim JH, et al. The effect of MLC speed and acceleration on the plan delivery accuracy of VMAT. 
Br J Radiol. 2015; 88(1049): 20140698, doi: 10.1259/ bjr.20140698, indexed in Pubmed: 25734490.

25. Prendergast BM, Fiveash JB, Popple RA, et al. Flattening filter-free linac improves treatment delivery efficiency in stereotactic body radiation therapy. J Appl Clin Med Phys. 2013; 14(3): 4126, doi: 10.1120/jacmp.v14i3.4126, indexed in Pubmed: 23652246.

26. Boda-Heggemann J, Mai S, Fleckenstein J, et al. Flatteningfilter-free intensity modulated breath-hold image-guided
SABR (Stereotactic ABlative Radiotherapy) can be applied in a 15-min treatment slot. Radiother Oncol. 2013; 109(3): 505-509, doi: 10.1016/j.radonc.2013.09.014, indexed in Pubmed: 24128805.

27. Scorsetti M, Alongi F, Castiglioni S, et al. Feasibility and early clinical assessment of flattening filter free (FFF) based stereotactic body radiotherapy (SBRT) treatments. Radiat Oncol. 2011; 6: 113, doi: 10.1186/1748-717X-6-113, indexed in Pubmed: 21910868. 